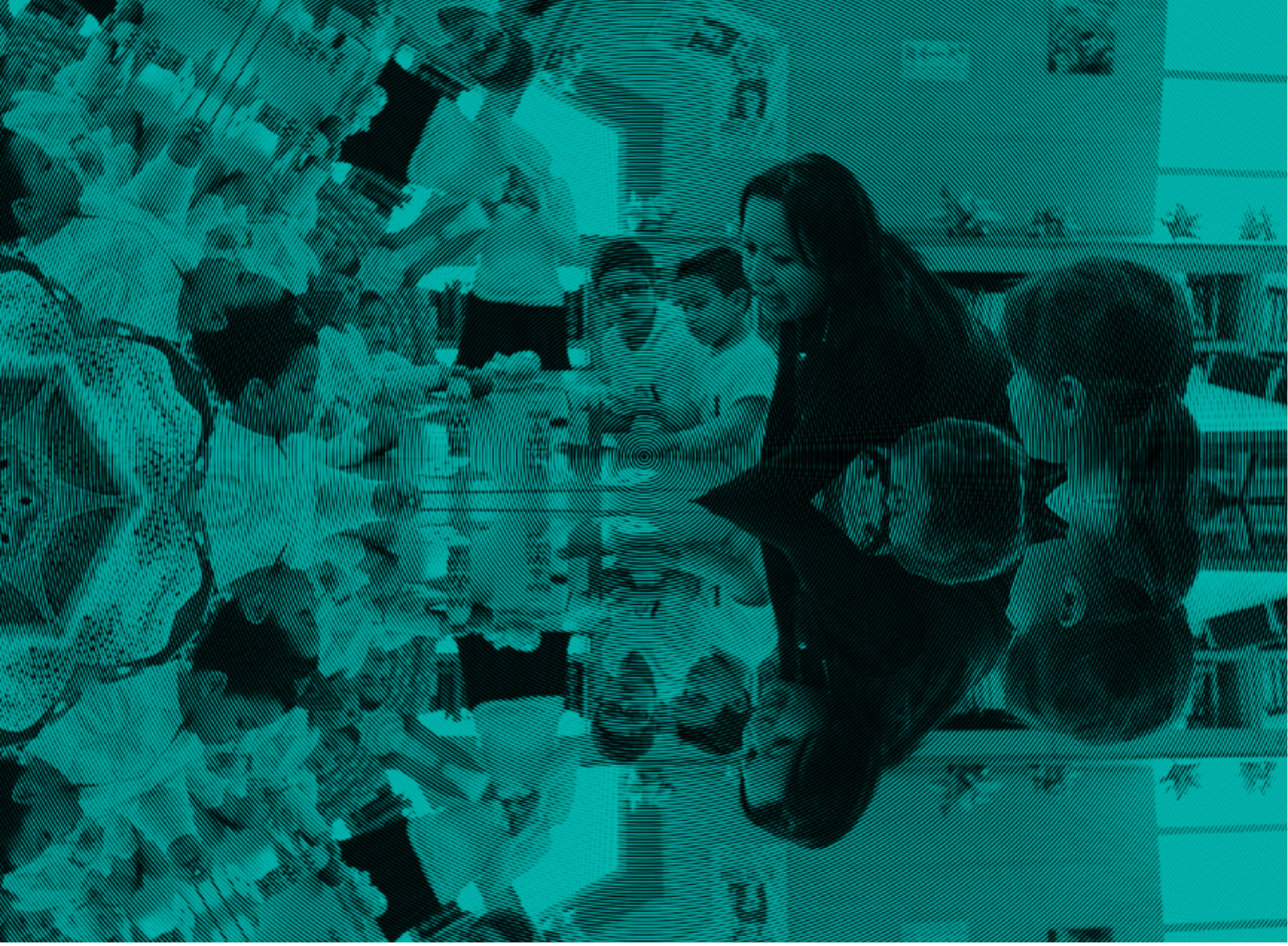

\title{
La convivencia escolar, un camino hacia la cultura de la paz
}

School of Life, a path to peace culture

Escola da Vida, um caminho para a cultura de paz

Esther Lucía Malagón Buitrago María Elizabeth Mateus Gómez Sonia Elena Gómez Carrillo 


\section{Esther Lucía Malagón Buitrago 1}

María Elizabeth Mateus Gómez 2

Sonia Elena Gómez Carrillo 3
1. Especialista en Gerencia de Proyectos Educativos UCC; Especialista en Educación y Gestión Ambiental. Ingeniera Forestal, Universidad Distrital Francisco José de Caldas. Docente IEM Guillermo Quevedo Zornoza, área Técnica; correo electrónico: galuben13@yahoo.com

2. Especialista en Gerencia de Proyectos educativos UCC. Licenciada en Dibujo Técnico UPN; Docente IEM. Técnico Industrial Zipaquirá, área de Tecnología e informática; correo electrónico: elmagovar26@hotmail.com

3. Especialista en Gerencia de Proyectos educativos UCC. Licenciada en Ciencias Sociales UPN; Docente IEM. Liceo Integrado Zipaquirá, área de Sociales; correo electrónico: Sonyhel@gmail. com
Fecha de recepción: 18 de abril de 2016 / Fecha de aceptación: 16 de septiembre de 2016

\section{Resumen}

El presente artículo expone la investigación realizada en una institución educativa del municipio de Zipaquirá, donde se pretendió identificar los factores de agresión y violencia que afectan la convivencia escolar, para elaborar una propuesta pedagógica que promueva la Cultura de la Paz; el interés partió de una necesidad de la institución y de lo exigido por las normas educativas, especialmente la Ley 1732 de 2014, que establece la Cátedra de la Paz. En la recolección de información se empleó la observación directa y la aplicación de encuestas al $35 \%$ de estudiantes de octavo y noveno.

Palabras clave: Propuesta pedagógica, convivencia escolar, cultura de paz, violencia escolar.

\section{Summary}

This article presents the research carried out in a school of Zipaquirá, where it is intended to identify the factors of aggression and violence that affect its school life in order to make a pedagogical proposal that promotes the Culture of Peace. It started from the need of the institution and the requirement of educational laws, especially Law $1732 / 2014$ that establishes the subject of Peace. For gathering information, it was used direct observation and surveys were applied to $35 \%$ of Eighth and Ninth graders students.

Keywords: Pedagogical proposal, school life, culture of peace, school violence.

\section{Resumo}

Este artigo apresenta pesquisa em uma instituição de ensino do município de Zipaquirá, que se destinava a identificar os fatores de agressão e violência que afetam a vida escolar, para desenvolver uma proposta pedagógica que promove a cultura de paz; o interesse veio de uma necessidade para a instituição e os requisitos das normas educacionais, em especial a Lei 1.732 de 2014, que estabelece o Departamento de Paz. Recolha de informação sobre a observação direta ea realização de estudos de $35 \%$ dos oitavo e nono foi usado.

Palavras chave: Proposta pedagógica, vida escolar, cultura da paz, violência escolar. 


\section{Introducción}

En la actualidad la convivencia juega un papel fundamental en el proceso de enseñanza, desde su función de fortalecer en los educandos los valores, principios y normas necesarios para vivir en comunidad y así lograr una cultura de paz. En los últimos años el fenómeno de violencia en el ámbito escolar ha sido tema de constante debate, ya que su ejercicio se ha incrementado en las últimas décadas, convirtiéndose en una problemática social abordada desde diferentes concepciones metodológicas y teóricas que buscan caracterizar, conceptualizar y/o solucionar este flagelo (Murcia, 2004).

De acuerdo con Rodríguez (2013) la violencia como fenómeno social es resultado de comportamientos, actitudes y relaciones disímiles entre los individuos que, con su actuar, infringen, causan lesión, daño o sufrimiento a alguno o varios miembros de la comunidad educativa; lo cual tiene como consecuencia un ambiente escolar difícil, tenso y estresante que puede causar más violencia. En Colombia las agresiones de tipo físico, verbal, psicológico, virtual y emocional al interior de las instituciones, son protagonizadas por directivos, docentes, padres, acudientes y estudiantes, siendo estos últimos los que presentan índices más altos y frecuentes (Palomero y Fernández, 2001).

Atendiendo a esta problemática, el Ministerio de Educación Nacional plantea una política hacia la consolidación de una cultura de paz que emane de la escuela, como agente responsable de: "enseñar a niños y jóvenes a vivir juntos en armonía y a convivir con base en el respeto a las diferencias y los derechos de los demás" (Ministerio de educacion nacional, s.f, p. 1). La escuela debe fomentar en niños y jóvenes el desarrollo de valores, actitudes, comportamientos y habilidades que promuevan la no violencia, en el marco del respeto y cumplimiento de los Derechos Humanos; además, debe planear e implementar estrategias orientadas hacia el cambio de actitudes violentas por relaciones pacíficas que posibiliten la construcción de una cultura de paz (García, 1998).

La violencia es una manifestación tan compleja que no está conformada solamente por los hechos concretos que la expresan, sino que a ellos subyacen emociones y valores (Prieto, Carrillo y Jiménez, 2005, p. 1031). El inadecuado uso de esas emociones genera violencia escolar, siendo la inteligencia emocional una habilidad que permite su adecuado manejo y, por tanto, la gestión de las mismas en el ámbito escolar se presenta como propuesta para mejorar la convivencia y consolidar una cultura de paz.

La presente investigación diagnóstica parte de lo observado en la Institución Educativa Municipal Guillermo Quevedo Zornoza, del municipio de Zipaquirá (Cundinamarca), que cuenta con una notoria problemática de convivencia escolar, con agresiones de todo tipo durante el desarrollo de la jornada que reflejan una escasa presencia de valores. Esta situación se materializa en actitudes hostiles hacia el entorno y en la mala relación entre compañeros, hechos que impiden una verdadera cultura de paz. Parte de la solución depende de la puesta en marcha de un proyecto que promueva la sana convivencia desde valores como el respeto, la tolerancia y la responsabilidad social.

El objetivo principal de esta investigación es identificar los factores de agresión y violencia que afectan los procesos de convivencia escolar, para diseñar una propuesta pedagógica que promueva una cultura de paz en la Institución Educativa Municipal Guillermo Quevedo Zornoza de Zipaquirá. Así como identificar las acciones pedagógicas adelantadas frente a los problemas de convivencia que influyen en la construcción de una cultura de paz, determinando los comportamientos, actitudes y relaciones que afectan la convivencia.

\section{Fundamentación teórica}

En primer lugar, la presente investigación aborda el concepto y fenómeno de la educación como realidad social y compleja, como ámbito en el cual se evidencian diversos problemas que merecen ser afrontados; luego se analiza la convivencia en el ambiente escolar, los principales factores que la afectan, la violencia y sus formas; después se fundamenta la necesidad de la educación para la paz, entendida como propuesta para fortalecer la convivencia, para luego anotar las principales teorías que han aportado a la investigación y educación para la paz, ofreciendo postulados de solución a la problemática de la violencia, útiles para desarrollar la construcción de una propuesta pedagógica que promueva y consolide la cultura de paz. Convivencia y cultura de paz son vistos como conceptos sociales, de construcción y de mejoramiento, que se enseñan y se aprenden (Ministerio de educacion de Chile, s.f.).

\section{La educación proceso de transformación}

Dependiendo de la disciplina y del contexto la educación ha tenido varias interpretaciones a lo largo de la historia; para el desarrollo del presente proyecto se retoma la idea de la educación como medio de transmisión y conservación de la herencia cultural, 
a través de la enseñanza de un sistema de valores y sentimientos que regulan el comportamiento y pautas del ser humano (Reyes, 2013). Así, la educación es un proceso que tiende a formar al individuo, fortaleciendo sus habilidades y capacidades para actuar conscientemente frente a las diversas situaciones de la vida. En el ámbito de la educación, y con el ánimo de cumplir con sus premisas teóricas, la escuela y las acciones pedagógicas que en ella se orientan juegan un papel clave, tienen unos objetivos y roles específicos que las configuran como ejes centrales en el proceso de formación de los niños y jóvenes. La escuela es el medio de socialización por excelencia, en ella el estudiante no solo aprehende una serie de conceptos y valores sino que los vive en la cotidianidad, siendo capaz de convivir respetando la diferencia, al otro y al entorno, emprendiendo las tareas de cambio y

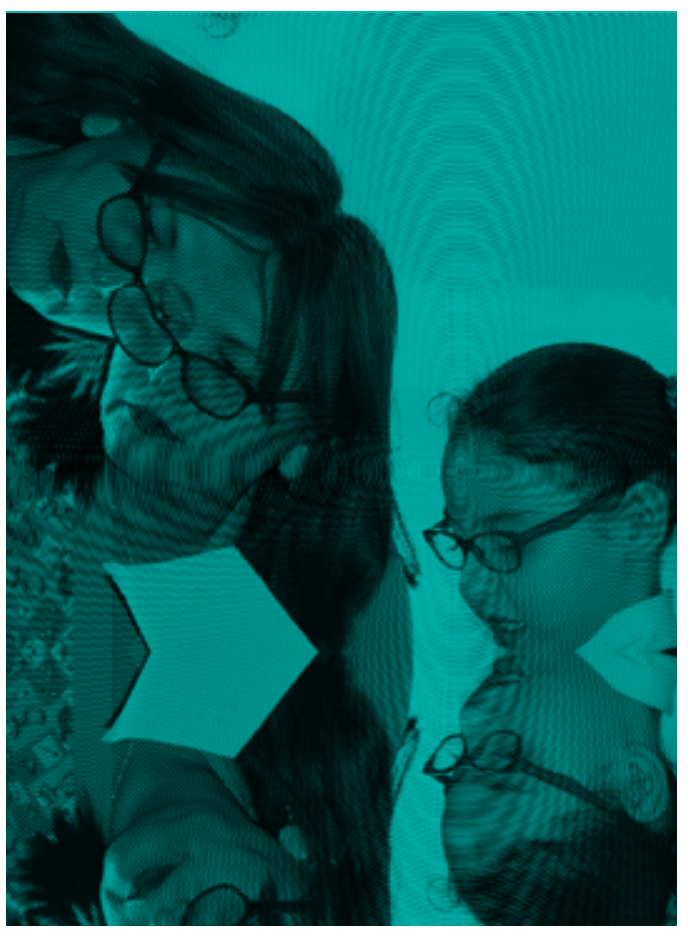
Violencia en el ámbito escolar

En el contexto social y en el ámbito escolar se evidencia un detrimento frente a la práctica de valores relacionados con el respeto de las normas, de la diversidad, de la pluralidad, de la diferencia, de la comunicación; hecho que se manifiesta en actos de violencia de toda índole que afectan negativamente la convivencia de la comunidad educativa, y que está ligado a factores como los sociales/ambientales, los relacionales, los escolares, familiares y personales (Perez, 2005). Al tiempo, se destacan ciertas conductas antisociales en los centros educativos y que son foco de violencia, tales como: Disrupción en las aulas, Indisciplina, vandalismo y daños materiales (Moreno, s.f.) transformación de la realidad.

\section{Convivencia en el ámbito escolar}

En última instancia la convivencia tiene que ver con:

La capacidad de las personas para establecer relaciones sociales y humanas de calidad, fundamentadas en la tolerancia y en el respeto de los demás. Esa calidad de las relaciones individuales y sociales se define no solo desde referentes, éticos, culturales y normativos, sino también desde competencias, habilidades y capacidades de los individuos para interactuar constructivamente (MEN, 2010).

La convivencia en el ámbito escolar, entendida como el entramado de relaciones e interacciones que se dan entre los miembros de la comunidad educativa, implica establecer procesos de comunicación, sentimientos, valores, actitudes, roles, status y poder. Este entramado de relaciones se ve afectado por: la indisciplina, los conflictos interpersonales, el maltrato entre compañeros, la irrupción en el aula, el vandalismo y la pérdida de valores (Ayuntamiento de LEIOA).

\section{La teoría tricerebral y la violencia}

La teoría tricerebral expresa que en cualquier grupo social siempre se presenta alguien que mande, que dirija; este tipo de persona pertenece al llamado subgrupo oficial; también alguien que critica y cuestiona, es decir los llamados subgrupo anti-oficial u opositores frente al primer grupo. Además, hay una gran mayoría que no está ni a favor ni en contra y es el llamado subgrupo oscilante o disponible, el cual fluctúa entre el grupo oficial y el anti-oficial según la conveniencia. Los tres subgrupos se disputan el poder sobre los medios de reproducción y convivencia, y en esta búsqueda pueden tener comportamientos positivos o negativos, proporcionales o desproporcionales (Gregori, 2002).

La lucha por alcanzar satisfacción desata una competencia violenta en la que el subgrupo oficial se caracteriza por buscar ávidamente el poder, persigue depredar y sacar el máximo de los demás con el mínimo riesgo; en esa búsqueda se dan tres tipos de violencia: la primordial, original, que en general está legalizada por el subgrupo oficial camuflada bajo la figura de estado o mercado; la violencia del grupo anti-oficial, que reacciona a la violencia uno y 
es tildada de ilegal y terrorista; y la del grupo oscilante, que sería la violencia callejera (Gregori, 2002).

\section{Educación para la paz}

Atendiendo a la actual situación, el Ministerio de Educación Nacional planteó una política hacia la consolidación de una cultura de paz cuyo eje principal sea la escuela, como agente responsable de: "enseñar a niños y jóvenes a vivir juntos en armonía y a convivir con base en el respeto a las diferencias y los derechos de los demás" (MEN, p. 1). A nivel mundial se ha generalizado la necesidad y el interés de educar para la paz; un concepto que a lo largo de la historia se ha relacionado con el de la guerra, su naturaleza y dimensión han variado de acuerdo al contexto económico, político, social y cultural.

La paz puede ser pensada, sentida y percibida desde diferentes puntos de vista (Muñoz, 2001), esto significa que las condiciones de cada época y las tendencias de pensamiento han planteado distintas teorías acerca de la paz como alternativa para solucionar los episodios de violencia y forjar una sociedad pacífica y justa, basada en el respeto de los Derechos Humanos, de la dignidad y la diversidad, fomentando el diálogo y transformando la realidad. Por ello, a continuación se mencionan los principales aportes de la investigación para la paz (Xexús, 2003), por las contribuciones significativas que realizó para el presente de la educación para la paz y la promoción de la cultura de paz.

\section{Investigación de la paz para "la cultura de paz"}

\section{La paz imperfecta (Muñoz Francisco, 2001)}

La "Paz imperfecta" de Muñoz busca abordar el tema desde la perspectiva del conflicto, refiriéndose a todas las experiencias en las cuales ha sido posible regular las situaciones pacíficamente. La llamó imperfecta porque considera las particularidades inherentes a la especie humana que llevan a que sea conflictiva, hecho que se hace evidente en el choque entre el entramado de intereses y necesidades individuales y la variedad de posibilidades que ofrece la sociedad; también buscaba romper con otras ideas que enmarcan la paz como algo perfecto, utópico e infalible, alcanzable en otro mundo con la mediación de los dioses, fuera del alcance de los humanos.
La paz imperfecta es un proceso inacabado y parte de argumentos como los de Gandhi: "No hay camino para la paz, la paz es el camino", para ver la paz como un principio que se construye cotidianamente. Esta teoría no solo busca la regulación, gestión y resolución de los conflictos de forma pacífica, sino que está relacionada con el poder como capacidad para transformar la realidad y medio para enfrentar los conflictos, buscando un equilibrio entre las tres esferas del poder: integrativa (amor, solidaridad, cooperación), destructiva (guerra y violencia) y productiva (económico).

\section{Teoría de los conflictos, la paz positiva, la paz negativa de Johan Galtung}

El aporte teórico de Galtung se centra en tres ejes: paz, conflicto y violencia, considerando las relaciones entre ellos, especialmente entre los dos últimos; desde su perspectiva la regulación del conflicto por medios no violentos es clave para lograr la paz, de tal manera que resulta fundamental analizar el papel de la cultura y de las estructuras sociales como generadoras de conflictos y de manifestaciones violentas (García, 2009, pp. 154-159). Galtung parte de la premisa de que a lo largo de la historia los conflictos son inherentes a la condición humana y de que, como tal, han contribuido tanto a su progreso como a su destrucción, siendo esta última una causa importante de la total deshumanización (Percy, 2009). Por eso es necesario abordarlo, estudiarlo y analizarlo desde su origen y naturaleza, para teorizarlo en beneficio de la humanidad.

\section{Violencia directa, violencia cultural y violencia estructural}

Para Galtung existen tres formas de violencia que se deben analizar para buscar estrategias de paz:

- Violencia directa: Los efectos visibles de la violencia; un acontecimiento que infringe o causa daño o lesión a alguien y que es visible; en este grupo están todas aquellas manifestaciones cotidianas de agresión (García, 2009, p. 129).

- Violencia cultural: Aspectos de la cultura materializados por medio de la religión, la ideología, el arte el lenguaje y las ciencias, que con sus diferentes manifestaciones legitiman la violencia directa y estructural (García, 2009, p. 130).

- Violencia estructural: Violencia indirecta generada por la injusticia y la desigualdad, es consecuencia de la propia estructura social, y puede darse dentro de ella o entre el conjunto de sociedades (alianzas, relaciones entre estados) (García, 2009, p. 130). 


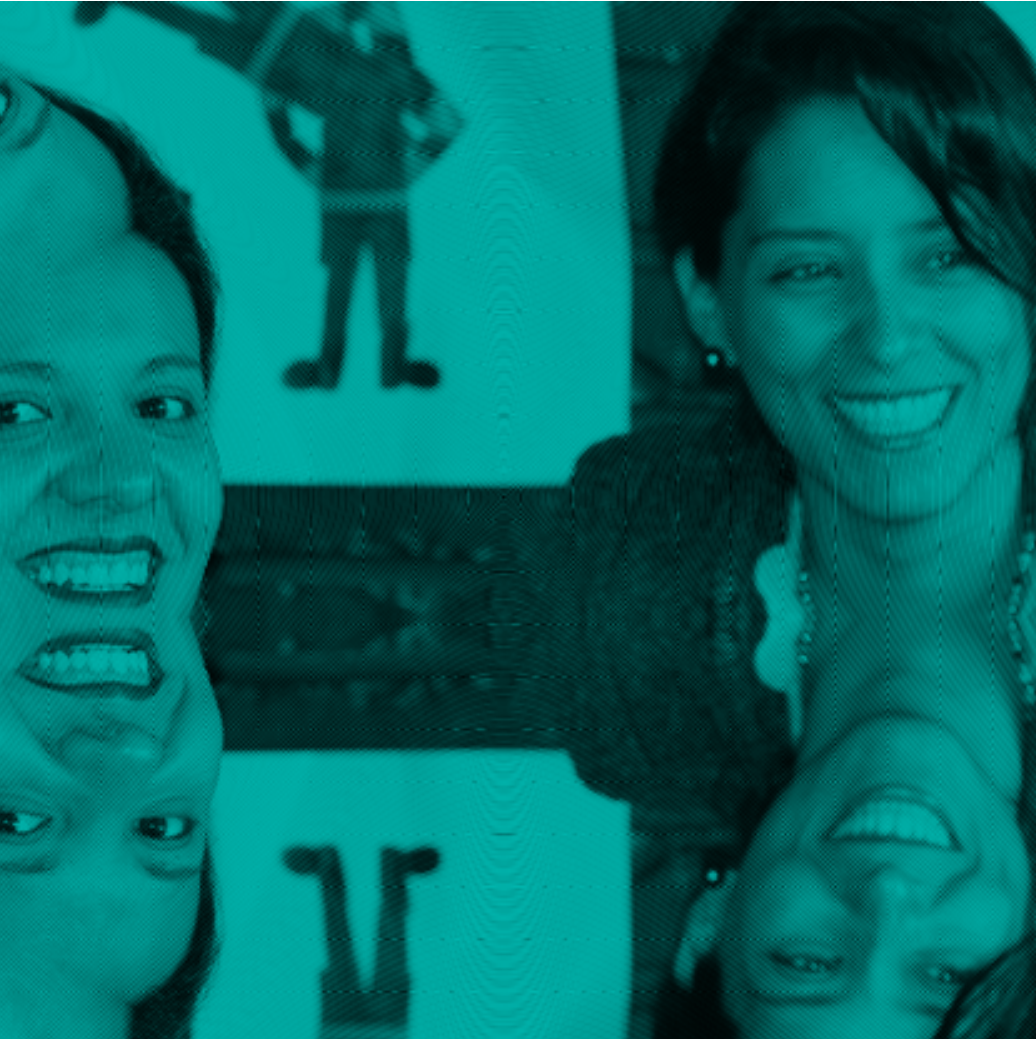

La paz para Galtung se logra cuando los conflictos se solucionan pacíficamente, cuando se coloca en práctica la habilidad para resolverlos de la mejor manera; ésta se debe entender como un proceso dentro de un contexto, una estructura y una organización, es decir que la sociedad requiere de un sistema donde haya una cultura de paz que garantice la resolución de los conflictos por medios no violentos (Percy, 2009). Desde esta perspectiva, la forma más acertada de construir una cultura de paz en las instituciones escolares es generar una conciencia de cambio a partir del compromiso de sus integrantes para emprender, en conjunto, acciones preventivas de los conflictos y de la violencia.

\section{Proporcionalismo}

El Proporcionalismo es planteado Gregori como una nueva justicia tríadica para regular la convivencia, entendiendo que la proporcionalidad es algo innato en los tres tipos de cerebros: para el cerebro derecho es dada por el sentimiento intuitivo-místico de ética, moral, justicia, humanismo, armonía, belleza o arte; para el cerebro izquierdo esta percepción se da a partir de la ley matemática de la media y extrema razón: el punto que las divide es la "sección áurea" o "Punto de Oro", también conocido como la divina proporción, proporción áurea, razón áurea o número de oro porque es un estándar que se repite universalmente; el cerebro central busca la proporcionalidad mediante un esfuerzo organizativo de imponer instituciones reguladoras y preservar el estado de derecho con sus leyes, códigos penales y justicia.

Para el Proporcionalismo no hay nada fijo, estable, inmóvil o lineal, todo ondula, oscila, fluye, se mueve en transformación, con rumbos y resultados más probabilísticos (menos caos) o menos probabilísticos (más caos). Así, el Proporcionalismo se define como una propuesta ético-numérica de vida y justicia social basada en la matemática de la media y la extrema razón, que supone, en todo, medidas aproximadas del 38\% y $62 \%$. Este es el punto de oro conocido como sección áurea (Gregori, s.f.). Cuanto más cercanos al punto de oro, más tendremos paz, disfrute y cooperación social, entre más lejanos, más competencia, guerra, infelicidad, crisis y explosión social.

\section{Hacia la promoción de una cultura de paz}

La UNESCO define la cultura de paz como un conjunto de valores, actitudes, tradiciones y estilos de vida basados en el respeto a la vida y en la práctica de la no violencia a través de la educación, la libertad de expresión, la justicia, la democracia, la tolerancia, la solidaridad, el pluralismo, la diversidad cultural y el entendimiento (UNESCO, 1998). La cultura de paz debe propender principalmente por practicar la no violencia y rechazar la violencia física, sexual, psicológica, económica y social en todos sus aspectos, en particular cuando afecta a los más débiles como niños y adolescentes.

\section{Metodología}

El proyecto parte de las necesidades del entorno, del horizonte institucional y de las exigencias frente al desarrollo de la convivencia; está enmarcado en las normas en educación y la Ley 1732 de 2014, que expone la necesidad de trabajar la cultura de paz en las instituciones educativas. Su desarrollo tuvo en cuenta un proceso de investigación que implicó la recolección y observación de información, datos, hechos, casos, y su posterior proceso, análisis e interpretación. Esto permitió comprender y describir la situación real de convivencia escolar en la Institución Educativa Guillermo Quevedo Zornoza, para fomentar la cultura de paz con la aplicación del Ciclo Cibernético de Transformación (CCT). El proceso de recolección de la información empleó inicialmente la 
observación directa, que se registró en una ficha de observación (instrumento), en segundo lugar se aplicaron encuestas al treinta por ciento de los estudiantes de los grados octavo y noveno (población de estudio), los resultados obtenidos se analizaron y triangularon para finalmente obtener las conclusiones.

\section{Resultados}

El proceso de análisis de datos y triangulación tomó en cuenta los estamentos de estudiantes y docentes; y agrupó distintas categorías: convivencia escolar, cultura de paz y propuesta pedagógica. A continuación se presentan los resultados más relevantes.

\section{Categoría convivencia escolar}

Esta categoría agrupó lo relacionado con la convivencia al interior de la institución, su dinámica y los procesos que se llevan a cabo. Al analizar cuáles son las situaciones de conflicto que afectan la convivencia escolar (Graficas 1 y 2), estudiantes y docentes señalaron que este tipo de actos se da más frecuentemente entre estudiantes ( $76 \%$ de los estudiantes y $88 \%$ de los docentes); y un bajo porcentaje opinó que se da entre estudiantes y docentes $(5 \%$ de los estudiantes y $12 \%$ de los docentes), lo que permite concluir que la interacción alumno-docente es buena, imperando el respeto mutuo (Actores del conflicto).

Los estudiantes desconocen el proceso a seguir frente a un conflicto; aproximadamente el $70 \%$ de ellos acude a algún miembro de la comunidad educativa para atender la situación, pero el $30 \%$ restante se queda callado, lo que impide solucionar el conflicto y puede desencadenar un episodio de violencia. Las situaciones de conflicto con el entorno no son tomadas en cuenta por los docentes, y tan solo un $19 \%$ de los estudiantes las identifica; sin embargo, teniendo en cuenta lo registrado en la ficha de observación, frecuentemente los estudiantes arrojan basura al piso y destruyen la infraestructura del plantel al dañar los pupitres o rayar las paredes.

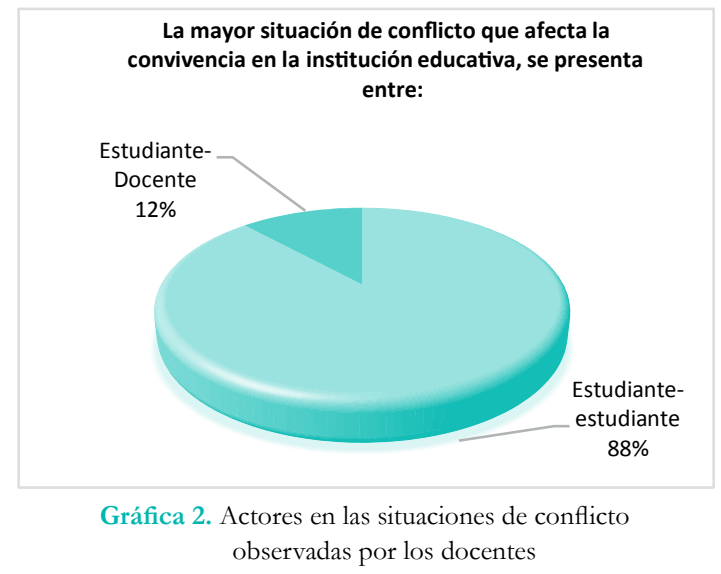

En cuanto al cumplimiento de las normas de convivencia, el manual de convivencia se presenta como una herramienta que no ha sido apropiada por la comunidad educativa; los docentes afirmaron que hay desconocimiento frente a los procesos y acciones pedagógicas correctivas a seguir en caso de conflictos o agresiones; los estudiantes corroboraron lo anterior: el $29 \%$ de ellos expresó que el manual de convivencia no es claro sobre lo que se debe hacer; el 23\% afirmó que presenta los casos de agresión pero que no son claros los procesos y acciones correctivas; y un 18\% dijo que posee unos procesos correctivos claros. Esta situación permite inferir la 
poca funcionalidad del manual de convivencia en la institución, o la falta de divulgación, implementación, apropiación y mejoramiento del mismo. Es necesario reflexionar acerca de su papel y reorientar su funcionalidad.

En el ejercicio de iniciativa y toma de decisiones por parte de los estudiantes, aspecto clave en el proceso de convivencia, se hizo evidente una debilidad en los canales y estrategias de comunicación, debido a que un $52 \%$ de ellos no manifiesta su opinión abiertamente porque teme no ser aceptado y por apatía; el resto lo hace

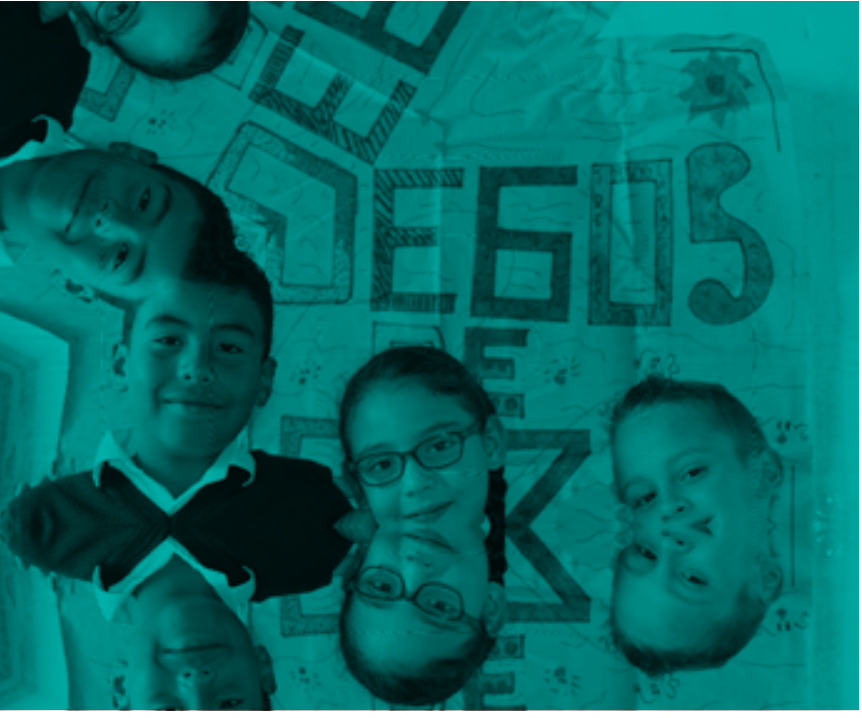

Categoría cultura de paz

Esta categoría tomó en cuenta las preguntas que aportan a la construcción de una cultura de paz, o que, para el caso del presente diagnóstico, dificultan este proceso. Los estudiantes y docentes tienen la percepción de que en el colegio se presentan con mucha frecuencia situaciones de agresión y conflictos (Gráficas 3 y 4); así, hay un porcentaje considerable de situaciones conflictivas que, a su vez, reflejan la carencia de valores como la justicia, la solidaridad, la dignidad y la armonía, hecho que dificulta los procesos de forma particular, ya sea con un profesor o un compañero, para evitar situaciones de burla. Lo anterior lleva a concluir que debe fortalecerse la comunicación en la convivencia escolar, especialmente la participación de los estudiantes en el liderazgo y toma de decisiones en la comunidad.

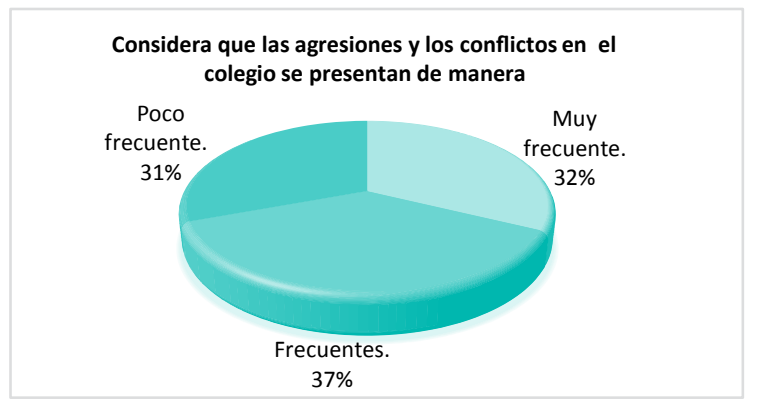

Gráfica 3. Frecuencia con la que se presentan situaciones de agresividad y conflictos según la percepción de los estudiantes

Los docentes y estudiantes encuestados identifican que las situaciones de conflicto más recurrentes entre los estudiantes son las faltas de respeto, que se expresan en agresiones físicas y verbales, insultos y amenazas (estudiantes $43,5 \%$ y docentes $76,4 \%$ ), todo esto desencadena altos índices de violencia. Las agresiones virtuales aparecen con un menor porcentaje con expresiones como: amenazas por redes sociales, acoso o ciberbullying, este menor índice de convivencia y la transformación de mentalidad, de actitudes y comportamientos que promuevan una cultura de paz; si se compara esto con la Gráfica 1, es evidente que los actores principales de los conflictos son los estudiantes, lo cual ratifica el ambiente de violencia que se vive a diario en la institución, que se materializa generalmente en agresiones de tipo físico o verbal.

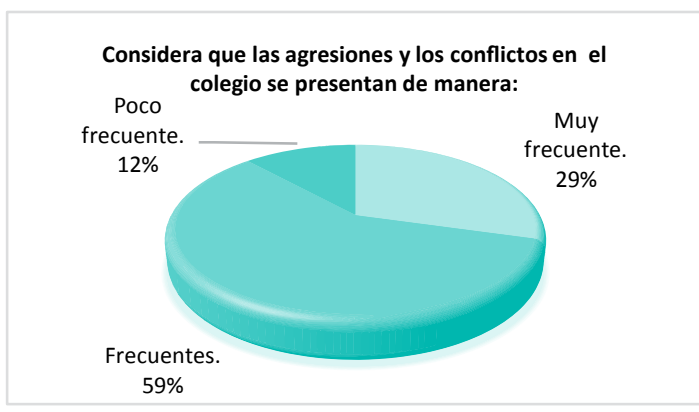

Gráfica 4. Frecuencia con la que se presentan situaciones de agresividad y conflictos

se debe al bajo acceso de los estudiantes a la tecnología, que a su vez se relaciona con su bajo nivel socioeconómico (el 46,8\% de estudiantes ven esta situación como poco recurrente).

El 32,3\% de los estudiantes perciben que el vandalismo es un hecho medianamente recurrente; para ellos esta situación no representa una agresión y es desde allí que no entiendan hechos como arrojar basura, rayar paredes y pupitres, dañar los muebles del 
colegio o desperdiciar agua, como una agresión hacia el entorno; esto refleja una falta de sentido de pertenencia y un consecuente menoscabo del cuidado y protección del medio ambiente; muchas de estas acciones son hábitos adquiridos en el hogar y ocupan un lugar en la vida en sociedad de los individuos. Un 41,2\% de los docentes sí identifican que el vandalismo es una situación medianamente recurrente, y un 29,4\% la entiende como recurrente.

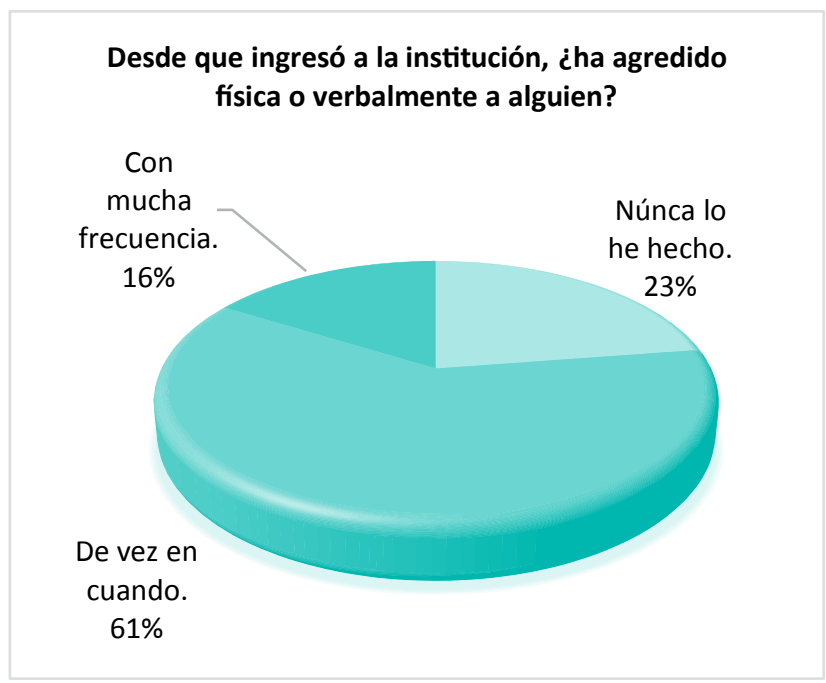

Gráfica 5. Frecuencia con la cual los estudiantes agreden a sus compañeros

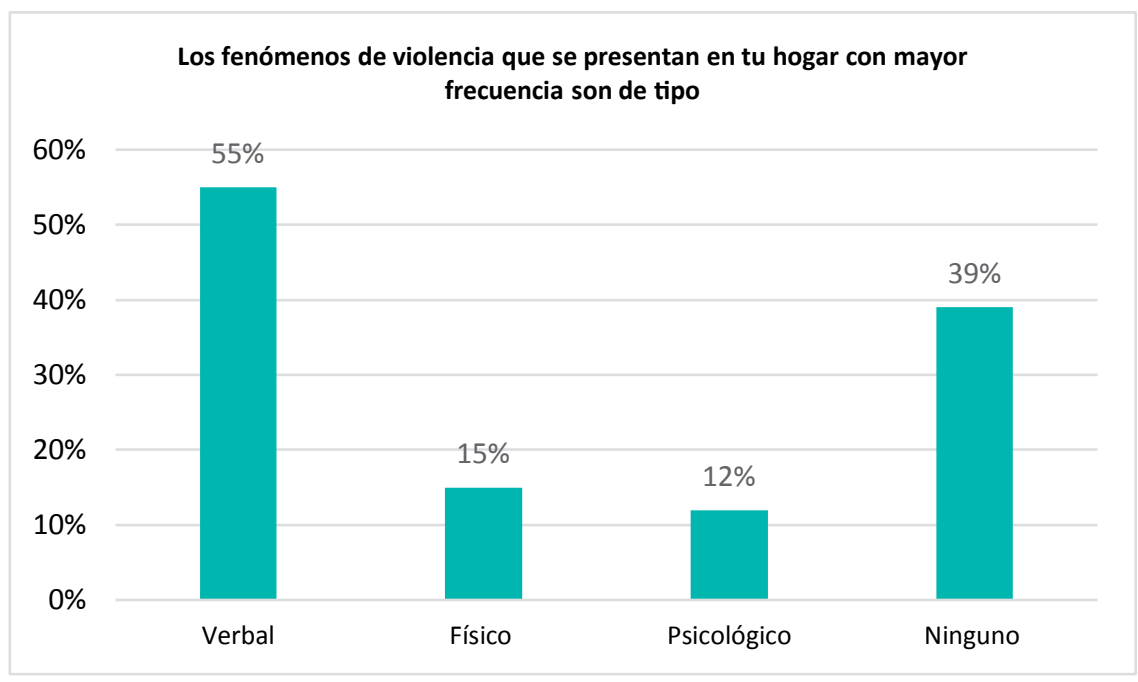

Gráfica 6. Manifestaciones de violencia en el hogar

En cuanto a la frecuencia de agresión, 61\% de los estudiantes encuestados ha agredido a otro compañero (Gráfica 5) de vez en cuando y un $23 \%$ nunca lo ha hecho; un $16 \%$ de los estudiantes reincide en situaciones agresivas de tipo físico o verbal, en muchos casos pueden incitar a otros a situaciones de violencia haciéndolos sus víctimas, llevándolos a situaciones de matoneo, hecho que 
dificulta los procesos de convivencia y la práctica de una cultura de paz.

Solo el 29\% de los docentes expresa que nunca se ha sentido agredido, mientras que un $29 \%$ considera haberlo sido en alguna oportunidad, ya sea por un estudiante o por un acudiente o padre de familia, y un bajo porcentaje ha tenido otro tipo de agresión. Cuando se presentan esas situaciones la mitad de los docentes afirmó no haber contado con apoyo de los directivos o de la Secretaría de Educación, 17\% dijo haber tenido ese apoyo y un 33\% expresó haber sido respaldado por alguna de las partes; esto hace evidente una debilidad por parte de las directivas, pues se permiten faltas de respeto de estudiantes y acudientes hacia los docentes; también destaca el hecho de que los profesores desconocen el funcionamiento del comité de convivencia municipal y la ruta de atención integral.

Cuando se piensa en los motivos por los cuales los estudiantes son agresivos en el colegio, se infiere que muchos de ellos aprenden este tipo de conducta en sus hogares (Gráfica O), donde en su mayoría se sienten agredidos verbal (55\%) y físicamente $(15 \%)$; pocos de ellos no viven situaciones de agresión familiar (39\%). Ello permite concluir que no se viven valores al interior de la familia y que su papel de corresponsabilidad en la educación de niños y jóvenes no está contribuyendo a forjar la cultura de paz.

\section{Categoría de propuesta pedagógica}

Con estas preguntas se buscó tener elementos que permitan identificar los gustos y preferencias de los estudiantes y docentes, para así considerarlos en el diseño de la propuesta pedagógica. Docentes y estudiantes reconocen que las actividades culturales y deportivas son espacios que permiten practicar la sana convivencia, y ese aspecto se tuvo en cuenta como estrategia para la construcción de la propuesta pedagógica. Los estudiantes expresan mayor interés hacia las actividades deportivas (68\%), seguidas de las actividades culturales (37\%); por su parte los docentes prefieren la actividades culturales $(64,7 \%)$ y luego las deportivas (53\%) (Gráfica 7 y 8).

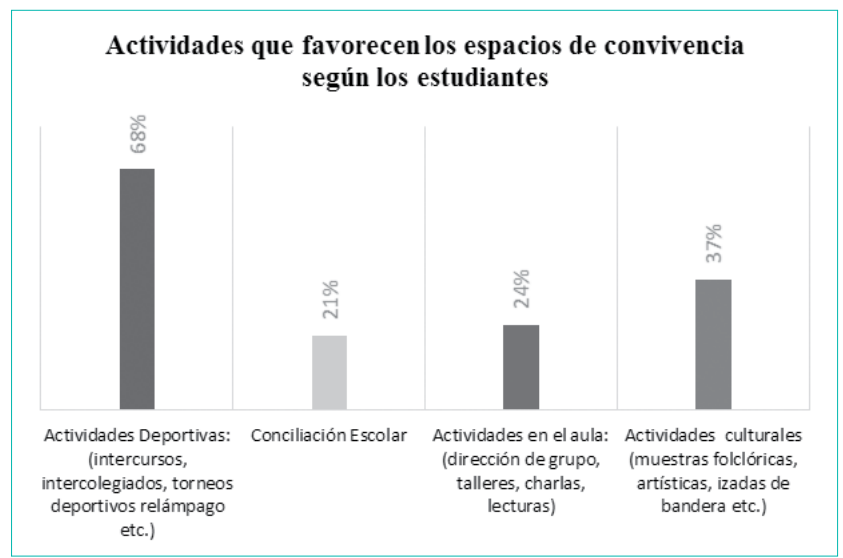

Gráfica 7. Actividades que favorecen los espacios de convivencia según los estudiantes

\section{Actividades que se considera favorecen los espacios de convivencia segun los docentes}

Actividades culturales (muestras folclóricas, artísticas, izadas de bander etc.)

Actividades en el aula: (dirección de grupo talleres, charlas, lecturas)

Conciliación Escolar

Actividades Deportivas: (intercursos, intercolegiados, torneos deportivos relámpago etc.)

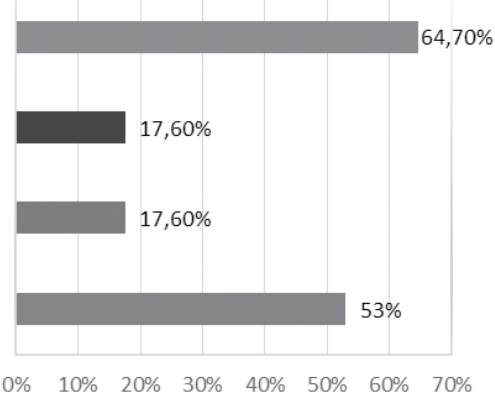

Gráfica 8. Actividades que favorecen los espacios de convivencia según los docentes 


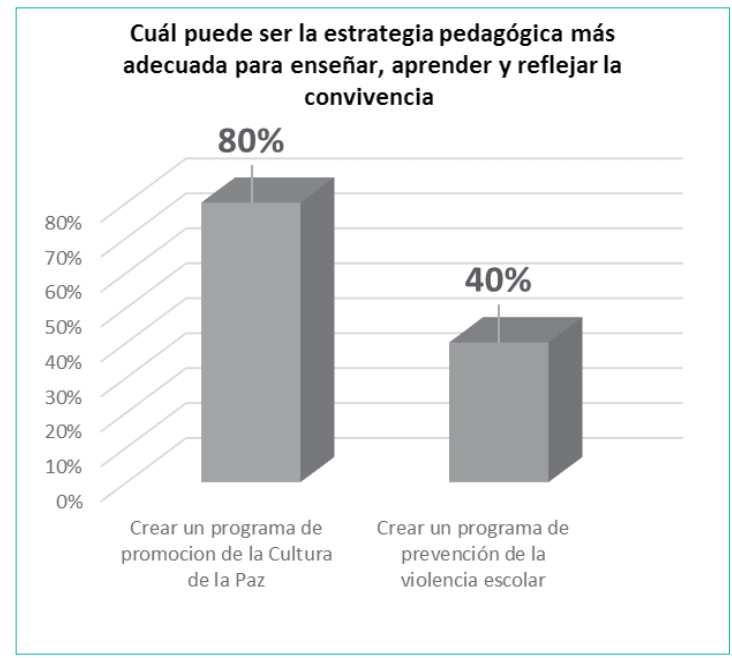

Gráfica 9. Estrategia pedagógica propuesta por los docentes

En su mayoría, los docentes opinan que la estrategia pedagógica más adecuada para enseñar, aprender y reflejar la convivencia es la creación de un programa de promoción de Cultura de Paz (80\%), hecho que se relaciona directamente con lo establecido en la norma frente al tema de la Cátedra de la Paz (Ley 1732 de 2014, Cátedra de la Paz; Decreto 1038 del 25 de Mayo de 2015, reglamentación de la Cátedra de la Paz), y un $40 \%$ prefiere un programa de prevención de la violencia escolar; ambas opciones pueden ser integradas en un único programa. Al tiempo, los estudiantes creen en su mayoría que las soluciones para trabajar la convivencia escolar parten de dialogar de manera efectiva, y proponen la realización de convivencias y campañas sobre la solución de conflictos; de igual forma, resaltan la importancia de promover valores como el respeto y la tolerancia, aspectos que también se convierten en insumos para la construcción de la propuesta pedagógica. Sin embargo, llama la atención sus demandas de tomar medidas correctivas eficientes frente a los agresores y atención de sus maestros (Gráfica 10).

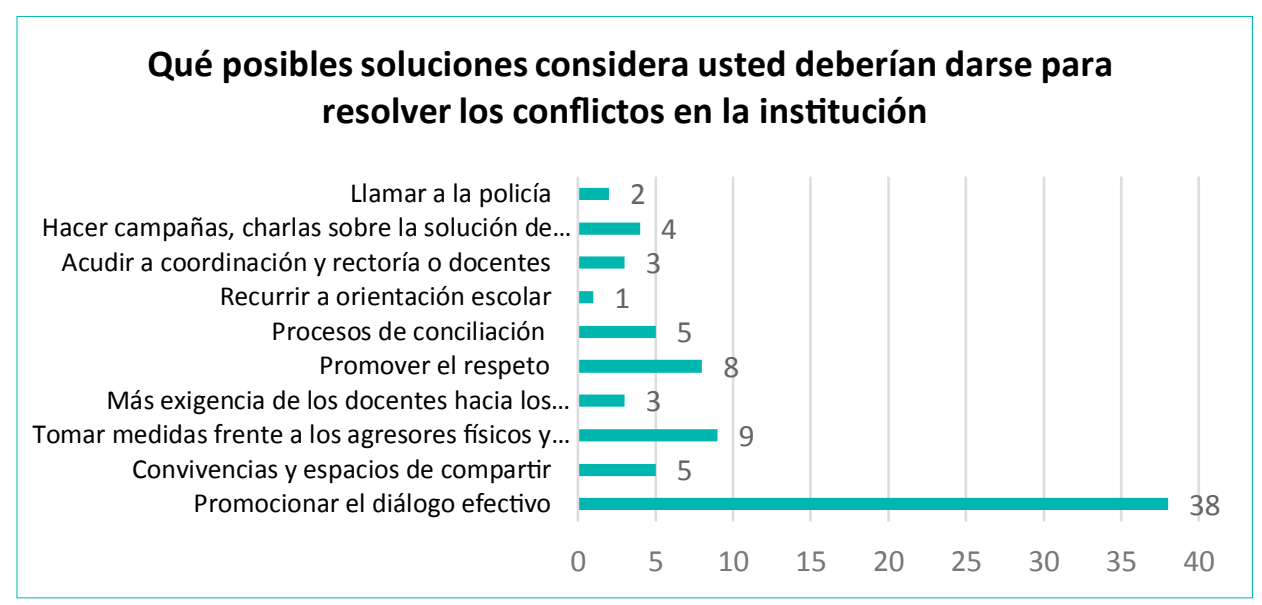

Gráfica 10. Posibles soluciones a los problemas de convivencia, propuestas de los estudiantes 


\section{Discusión de resultados}

Tanto en la ficha de observación como en los resultados de las encuestas se presentó un alto índice de agresividad en la institución, protagonizada generalmente por los alumnos. Este aspecto fue registrado inicialmente en la ficha de observación y corroborado a través de las encuestas. Las agresiones de tipo verbal y físico fueron problemas de convivencia destacados por estudiantes y docentes, observados por el grupo investigador, y registrados en la ficha de observación teniendo en cuenta los diferentes espacios y momentos, como las horas de descanso. A ellas se suma como elemento destacado, registrado y verificado en las encuestas, la actitud de los estudiantes frente al trabajo en equipo y la convivencia sana en la organización y participación de actos culturales y prácticas deportivas. Este aspecto es esencial en la planeación de la propuesta pedagógica, objeto de este proyecto.

En contraste con lo expuesto en el marco teórico, el diagnóstico realizado a estudiantes y docentes de la Institución Educativa Municipal Guillermo Quevedo Zornoza, permitió observar que tanto en la institución analizada como en el país las agresiones de tipo físico, verbal, psicológico, virtual y emocional al interior de las escuelas son protagonizadas por directivos, docentes, padres, acudientes y estudiantes; siendo el índice más alto el de las agresiones estudiante-estudiante (Palomero y Fernández, 2001); según lo expuesto por Gregori en la teoría tricerebral, siempre habrá un juego tríadico. El comportamiento de un subgrupo es positivo cuando toma en cuenta a los a los otros dos y alcanzan una división proporcional de derechos y deberes: gana-gana-gana, si esto se logra se puede hablar de convivencia y solución de conflictos. Docentes y estudiantes de la institución educativa tienen un desconocimiento frente a los procesos de convivencia reglamentados en la ley 1620 de 2013, debido a lo poco funcional del manual de convivencia, que debe ser actualizado, socializado y apropiado por parte de docentes, estudiantes y padres de familia.

Hay distintos factores que dificultan el alcance de una cultura de paz al interior de la institución educativa, como la agresividad, no solo entre estudiantes, sino para con el entorno mediante acciones de vandalismo, relacionadas con el ámbito social y familiar. Indiscutiblemente el primer órgano educativo en la sociedad es la familia, y la situación observada comprueba que los estudiantes enfrentan situaciones de violencia verbal y física en sus hogares, por lo que se puede concluir que la familia no está ejerciendo su función de corresponsabilidad en la educación de niños y jóvenes, y no contribuye a forjar la cultura de paz; la desintegración del grupo familiar, la dispersión de sus miembros y la desatención de los hijos contribuyen a la no cultura de paz.

\section{Conclusiones}

Las diferentes teorías, como la paz imperfecta de Muñoz, la teoría de la paz o el equilibrio de Valdéz y los aportes de Galtung, dan pautas para lograr una verdadera educación para la paz y cultura de paz, a partir de tres ejes básicos: paz, conflicto y violencia, y sus distintas relaciones; en el proceso de investigación diagnostica se abordó el conflicto desde su regulación y gestión, como una oportunidad para que se dé el diálogo, los valores, y la prevención de la violencia; la convivencia escolar se convierte así en un entramado de interacciones entre la comunidad educativa: estudiantes, docentes, directivos, padres, madres y acudientes, y el conflicto será una oportunidad de mejoramiento. Cuando en una institución, como la analizada, no se da esa relación en un marco de respeto mutuo, solidaridad y tolerancia, se generan gran cantidad de conflictos que desencadenan expresiones de violencia, afectando el clima escolar. Ese es el caso de la Institución Educativa Municipal Guillermo Quevedo Zornoza de Zipaquirá, donde los problemas de convivencia no permiten que se forje la cultura de la paz.

Los problemas se dan especialmente entre estudiante-estudiante, quienes comúnmente agreden y han sido agredidos por otro; las agresiones más frecuentes son las físicas y verbales, causadas por la intolerancia entre ellos, y el vandalismo. Cuando se presentan conflictos los estudiantes manifiestan que no cuentan con apoyo y que no informan la situación por miedo a que no les presten atención o a sufrir burlas; también expresan que los procesos de comunicación son deficientes para sortear conflictos, porque pocas veces se solucionan por medio del diálogo. Estos problemas de comunicación no solo son de la institución sino que se dan en las relaciones familiares; los jóvenes admiten que son frecuentemente sometidos en casa a agresiones verbales y físicas.

Partiendo de la realidad institucional verificada con el diagnóstico, es evidente la necesidad de construir una propuesta pedagógica que permita mejorar la convivencia escolar, brindando espacios de socialización guiados, donde el desarrollo de actividades pedagógicas enfocadas al deporte, la cultura y el arte, sean espacios de aprendizaje para enseñar, aprender y reflejar la sana convivencia escolar, facilitando a los estudiantes la posibilidad de practicar valores como el respeto, la tolerancia y el trabajo en equipo, generando cambios de mentalidad que se reflejen en actitudes y 
comportamientos socialmente favorables. Los docentes comparten esta perspectiva, pues piensan que la promoción de la cultura de paz puede contribuir a mejorar los procesos de convivencia.

Estos aspectos no son ajenos a los enunciados teóricos, pues autores como Reyes (2013) reconocen que los espacios educativos transmiten una herencia cultural, inculcan valores y facilitan regular el comportamiento. Esta postura es ratificada por instituciones como la Universidad Rafael Landivar (2010), cuando aseguran que el individuo debe actuar de manera consiente frente a diversas situaciones de la vida, y que ello exige soluciones creativas; eso es lo que se busca a través de la propuesta pedagógica: brindar a la comunidad educativa una oportunidad de crear soluciones pacíficas a sus diferencias, aprendiendo a aprender, a construir una cultura de paz.
La triangulación de resultados y un exhaustivo análisis permitieron comprobar que el diseño de una propuesta pedagógica, que promueva la cultura de la paz, es la estrategia más adecuada para mejorar la convivencia en la institución. Además, el cruce ascendente de las categorías con el marco teórico, permitió inferir que la propuesta pedagógica debe estar enfocada en una etapa de prevención del conflicto y la violencia, para lo cual es importante considerar la posición de los docentes, quienes en un $80 \%$ creen que la estrategia pedagógica más adecuada, entendiendo el contexto de la institución, es la creación de un Programa de promoción de la Cultura de Paz, mientras un $40 \%$ se inclina por un programa de prevención de la violencia escolar; ambas opciones pueden ser integradas en un único programa. 


\section{Referencias}

Ayuntamiento de LEIOA. (s.f.). Programas y estrategias para la Convivencia Escolar. Obtenido desde http://www.leioa.net/vive_doc/guia_convivencia_cast.pdf

Fisas, V. (2006). Cultura de pazy gestión de conflictos. Barcelona: Ediciones Unesco.

García Correa, A. (1998, Octubre 20). Un aula pacífica para una cultura de paz. Revista electrónica interuniversitaria de formación del profesorado, 1(1), p. 2.

García, V. H. (2009). La transformación de los conflictos por medios pacificos. Obtenido desde file://C:/Users/pearl_000/AppData/Local/ Microsoft/Windows/INetCache/IE/G0K ER7WQ /Dialnet-JohanGaltung-595158.pdf

Gregori, W. D. (2002). Construcción familiar escolar de los tres cerebros. Bogotá: Kimpres.

Gregori, W. D. (s.f.). Neuroeducación para el éxito. Bogotá.

Instituto de paz y conflictos, Departamento de historia antigua. (2001). La paz imperfecta. Granada. http://wdb.ugr.es/ fmunoz/index.php/ paz-imperfecta.html

MEN. (2010). Politica educativa para la formación escolar en la convivencia. Obtenido desde http://www.mineducacion.gov.co/1621/articles-90103_ archivo_pdf.pdf

Ministerio de Educación de Chile. (s.f.). La convivencia escolar. Obtenido el 12 de Diciembre de 2015, desde http://www.convivenciaescolar.cl/ index2. php?id_seccion $=3375 \&$ id_portal $=50 \& i d \_$contenido $=13803$

Moreno Olmedilla, J. M. (s.f.). Comportamiento antisocial en los centros escolares: una visión desde Europa. Revista Iberoamericana de Educación (18).

Muñoz Francisco. (2001). La paz imperfecta. Obtenido desde http://wdb. ugr.es/ fmunoz/index.php/paz-imperfecta.html

Murcia, F. V. (2004, Enero-Junio). Conflicto y violencia escolar en Colombia: lectura breve de algunos materiales escritos. Guillermo de okhan, 7.
Organización de los Estados Iberoamericanos para la Educación la Ciencia y la Cultura. (OEI). (2011). Educación y cultura de la pa\%: Obtenido desde http://www.oei.es/valores2/palos1.htm

Palomero Pescador, J. E., y Fernández Domínguez, M. R. (2001-Agosto). La Violencia Escolar, un punto de vista global. Revista interuniversitaria de formación del profesorado, 41, pp. 19-38.

Percy, C. C. (2009). Teoría de los conflictos de Johan Galtung. Pazy Confictos(2), pp. 1-22.

Pérez, V. (2005). Conflictividad escolar y fomento de la violencia. Revista iberoamericana de educación (38), 33-52.

Prieto Quezada, M. T., Carrillo Navarro, J. C., y Jiménez Mora, J. (2005-Octubre). La violencia escolar, un estudio en el nivel medio superior. Revista Mejicana de investigación educativa RMIE, 10(27).

Reyes, J. C. (2013). Educación, interdisciplinariedad y pedagogía. Revista Mexicana de Investigación Educativa, 2.

Rodríguez, H. (2013-Septiembre). La violencia en la escuela; elementos para su discusión como objetos de estudio. Obtenido el 01 de feberero de 2015, desde http://soda.ustadistancia.edu.co/enlinea/paginaimagenes/PRESENTACIONESyPONENCIAS/Memorias\%20Ponencias/Bogota/ Educacion $\% 20 y \% 20$ derechos $\% 20$ humanos/Mesa\%201\%20Septiembre $\% 2020 /$ Paola $\% 20$ Herrera $\% 20$ Rodriguez.pdf

Rodríguez, J. P. (2011). Educación y Cultura de Paz: Obtenido desde http:/ / www.oei.es/historico/valores2/palos1.htm

UNESCO. (1998). Manual del buen explorador e iniciativas de Cultura de Pa\%: Obtenido desde http://escolapau.uab.cat/img/programas/cultura/ manual_explorador.pdf

Valdéz Medina, J. (2009). Teoría de la paz o equilibrio. México: Edamex.

Xexús R, J. (2003). Educación para la Paz, su teoria y su práctica. Madrid: Popular. 